\title{
Relaciones polen-vegetación de algunos taxa de la estepa patagónica (Argentina)
}

\author{
Pollen-vegetation relationships of some taxa from the Patagonian steppe (Argentina)
}

LIDIA S. BURRY ${ }^{1}$, MATILDE E. TRIVI DE MANDRI, PATRICIA I. PALACIO \& MARÍA C. LOMBARDO

Departamento de Biología, Facultad de Ciencias Exactas y Naturales, Universidad Nacional de Mar del Plata, Funes 3250, 7600 Mar del Plata, Argentina, e-mail': lburry@mdp.edu.ar

\section{RESUMEN}

La aplicación de un análisis de regresión lineal simple a datos de lluvia de polen y de vegetación, en una zona de la Patagonia (Provincia de Chubut, Argentina) permitió relacionar cuantitativamente los porcentajes de lluvia polínica y de cobertura de la vegetación para los taxa Poaceae, Papilionoideae, tipo Senecio y Mulinum spinosum. Se obtuvo un buen ajuste de las rectas de regresión para cada uno de los taxa considerados, con coeficientes de correlación r de Pearson altos para $M$. spinosum $(0,82)$ y tipo Senecio $(0,81)$ y coeficientes algo menores en el caso de Poaceae $(0,66)$ y Papilionoideae $(0,61)$. Estas regresiones permitieron inferir la presencia o ausencia de polen regional y el valor predictivo de la presencia de polen en ausencia de la vegetación que lo produce. Al respecto, la lluvia de polen estudiada incluye el aporte regional (excepto Papilionoideae) aun cuando $M$. spinosum tiene una baja contribución. Esta sobrerrepresentación es atribuible al transporte de larga distancia, a través de los vientos que provienen del oeste. Estas relaciones cuantitativas nos permitirán extrapolar cambios vegetacionales pasados en estos taxa a partir de espectros de polen fósil para el área de estudio.

Palabras clave: polen actual, cobertura vegetal, regresión lineal simple.

\begin{abstract}
The use of simple linear regression to data of pollen rain and vegetation cover in Patagonia (Province of Chubut, Argentina) allowed us to develop quantitative relationships between modern pollen rain perecentages and vegetation cover for the taxa Poaceae, Papilionoideae, Senecio type, and Mulinum spinosum. The analysis revealed a good fit of data to regression lines for all taxa, with high Pearson $r$ coefficient values in the case of M. spinosum (0.82) and Senecio type (0.81), and somewhat lower values in the case of Poaceae $(0.66)$ and Papilionoideae $(0.61)$. These regressions allowed us to infer the presence or absence of regional pollen and predict the quantity of pollen in the absence of the vegetation producing it. In this regard, the studied pollen rain includes the regional contribution (except Papilionoideae), even though $M$. spinosum has a low contribution. This overrepresentation is attributed to long-distance transport associated to the westerly winds. These quantitative relationships will allow us to know past vegetational changes of these taxa from fossil pollen spectra in the studied area.
\end{abstract}

Key words: modern pollen, plant cover, simple linear regression.

\section{INTRODUCCIÓN}

Los estudios palinológicos permiten reconstruir la historia de la vegetación sobre la base del conocimiento de la dispersión-depositación del polen actual, considerando que existe una relación entre la vegetación y el polen que ésta produce (von Post 1918, 1967, Godwin 1934a, 1934b, Erdtman 1943, Fagerlind 1952, Davis 1963, Janssen 1970, Walker 1972, D'Antoni 1990). Dado que existen diferencias en la productividad, el transporte y la depositación del polen de las dis- tintas especies, la relación entre la lluvia de polen y la vegetación que la produce rara vez es de uno a uno (Faegri \& Iversen 1989). Además, las interacciones de los distintos taxa con los diversos factores ecológicos hacen que la relación polen-vegetación varíe en tiempo y espacio. En consecuencia, es necesario establecer relaciones cuantitativas entre la lluvia de polen y la abundancia de las especies productoras; éste es un paso crítico en la construcción de modelos de las relaciones polen-vegetación. 
La dispersión del polen actual en la Patagonia ha sido investigada por varios autores (D'Antoni 1990, 1991, Mancini 1989, Páez 1991). D’Antoni (1990) aplicó el método estadístico de análisis de factores a datos de lluvia de polen provenientes de siete transectos oeste-este, desde los piedemontes andinos hasta la costa atlántica, entre los 38 y $54^{\circ} \mathrm{S}$, y de un microtransecto en el cerro Domuyo $\left(37^{\circ} \mathrm{S}\right)$. Pudo diferenciar, a través de este análisis, las condiciones ecológicas de las provincias fitogeográficas del Monte, Patagónica y Subantártica, y además de comunidades freáticas e «impactos» humanos sobre el ambiente natural.

Con objeto de encontrar las relaciones cuantitativas entre la lluvia de polen y la cobertura de vegetación, se han utilizado técnicas de regresión, de modo de calibrar datos de polen en términos de datos de vegetación (Jackson 1994, Jackson et al. 1995). Inicialmente, se realizaron análisis de regresión en sitios únicos (Curtis 1959, Davis \& Goodlett 1960, Davis 1963), pero estudios posteriores analizaron sitios múltiples (Andersen 1970, Bradshaw 1981, Webb et al. 1981). Enfoques más recientes han utilizado el enfoque de máxima similitud (Parsons \& Prentice 1981, Prentice 1986).

Varios autores han ajustado la transformación polen-vegetación para lograr modelos más confiables (Andersen 1970, Webb et al. 1981, Bradshaw \& Webb 1985, Faegri \& Iversen 1989). En los estudios de Webb et al. (1981) y Bradshaw \& Webb (1985) se utilizaron técnicas de regresión para estimar la abundancia de plantas a partir de porcentajes de polen. Webb et al. (1981) utilizaron datos de lluvia polínica de sedimentos de lagos y datos de abundancia de árboles a partir de inventarios forestales. El cálculo de parámetros de regresión para once taxa, simplificó la descripción de cómo la relación polen-árbol difiere entre taxa. En el sur de las provincias de Chubut, Santa Cruz y Tierra del Fuego, D'Antoni \& Spanner (1993) usaron regresión múltiple para calibrar datos de lluvia polínica en términos de imágenes satelitales, lograron predecir los valores de reflectancia de la vegetación en el rojo e infrarrojo cercano, y derivaron los índices de vegetación: relación simple (SR), e índice verde normalizado (NDVI). La primera aplicación de esta metodología a datos polínicos estratigráficos se realizó para el perfil de meseta Latorre (Provincia de Santa Cruz, D'Antoni \& Schäbitz 1995), donde la calibración de datos de polen mediante información de sensores remotos, permitió realizar reconstrucciones de la vegetación durante el período postglacial.
El presente trabajo es el primero en Argentina que tiene por objetivo utilizar análisis de regresión lineal simple para establecer relaciones cuantitativas entre el polen y la cobertura vegetal de diferentes taxa de la estepa patagónica (Provincia de Chubut). El conocimiento de estas relaciones, ya sea a partir de observaciones de campo, datos de teledetección o una combinación de ellos, permitirá alimentar los parámetros de modelos computacionales predictivos de la vegetación. Los taxa para los cuales se construyeron modelos son: Poaceae, Mulinum spinosum, tipo Senecio y Papilionoideae.

\section{MATERIALES Y MÉTODOS}

\section{Área de estudio}

El presente trabajo se realizó en el sur de la Provincia de Chubut, a los $45^{\circ} \mathrm{S}$ y entre los $68^{\circ}$ $57^{\prime}$ O y $\operatorname{los} 71^{\circ} 26^{\prime} \mathrm{O}$ (Fig. 1). La Patagonia es una región de vegetación semidesértica o desértica (Soriano 1956). El clima es seco y frío, con heladas frecuentes durante todo el año; las precipitaciones son escasas en la zona oriental (e.g., 189 mm en Comodoro Rivadavia, $45^{\circ} 47^{\prime} \mathrm{S}, 67^{\circ} 30^{\prime}$ O) y central (e.g., $125 \mathrm{~mm}$ en Sarmiento, $45^{\circ} 33^{\text {, }}$ $\mathrm{S}, 69^{\circ} 04^{\prime} \mathrm{O}$ ) pero aumentan hacia el oeste (e.g., $500 \mathrm{~mm}$ en el límite con Chile, $45^{\circ} \mathrm{S}$ ). Los vientos son fuertes y predominan aquellos provenientes del oeste, especialmente en los meses de verano (Beeskow et al. 1987). Este estudio se extiende en los siguientes distritos fitogeográficos: central, caracterizado por estepas arbustivas de Chuquiraga y Nassauvia (Asteraceae); occidental, caracterizado por estepas mixtas de gramíneas y arbustos, con Stipa y Poa (Poaceae), Mulinum spinosum (Apiaceae), Adesmia (Fabaceae, Papilionoideae) y Senecio (Asteraceae), y subandino, caracterizado por una estepa de gramíneas en la que predomina Festuca pallescens (Poaceae) (Soriano 1956, Cabrera 1994).

\section{Muestreo y análisis}

Se realizaron censos de vegetación en 11 estaciones ubicadas en los distritos central, occidental y subandino mediante el método de la línea de intercepción introducido por Canfield (1941) y Mueller-Dombois \& Ellenberg (1974). La localización de los censos coincide con los sistemas fisiográficos (según Beeskow et al. 1987) ubicados a $\operatorname{los} 45^{\circ} \mathrm{S}$ y entre $\operatorname{los} 68^{\circ} 57^{\prime} \mathrm{O}$ y $\operatorname{los} 71^{\circ} 26^{\prime}$ O. En cada estación se tendieron tres líneas de 10 $\mathrm{m}$ de largo donde se registró la cobertura de cada 


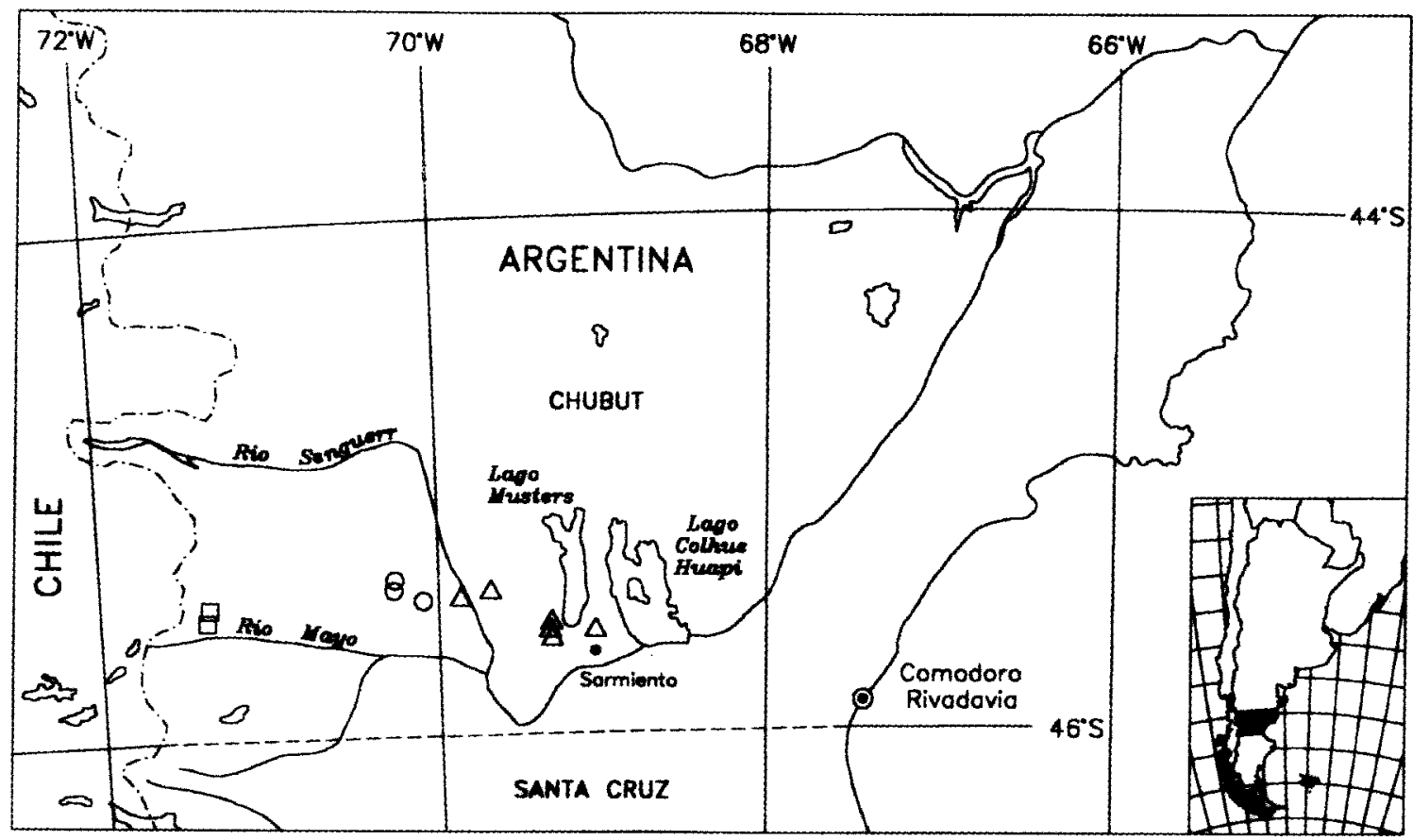

Fig. 1: Area de estudio y localización de las estaciones de muestreo de vegetación y polen y los distritos de los cuales provienen: distrito central $(\Delta)$, distrito occidental $(O)$ y distrito subandino $(\square)$ (Soriano 1956).

Study area and location of vegetation and pollen sampling sites: central district $(\Delta)$, occidental district $(O)$, and subandean district ( $\square$ ) (Soriano 1956).

taxón presente mediante una estimación de la longitud de la línea interceptada por la proyección perpendicular de las partes aéreas de cada individuo. Luego, se calculó el porcentaje relativo de cada taxón con respecto a la cobertura total de la vegetación.

Para la obtención de polen se tomaron tres muestras de sedimento superficial en cada estación de muestreo de vegetación. En el laboratorio se tomaron alícuotas de $20 \mathrm{~g}$ de las tres muestras de cada estación. Se agregaron tabletas de Lycopodium clavatum como marcador foráneo (Stockmarr 1971). La extracción de polen se hizo con métodos físicos y químicos (filtrado, $\mathrm{KOH}$, $\mathrm{ZnCl}_{2}$, HF y acetólisis) (Gray 1965, Faegri \& Iversen 1989). A partir de una suma polínica que varió entre 250 y 1.180 granos por muestra, y de acuerdo con métodos convencionales y de área mínima', se calcularon los porcentajes correspondientes a cada taxón polínico.

\footnotetext{
' Bianchi \& D'Antoni (1986) Depositación del polen actual en los alrededores de Sierra de los Padres (Prov. de Buenos Aires) Contribución al IV Congreso Argentino de Paleontología y Bioestratigrafía, Mendoza, Argentina. pp 16-27.
}

Se seleccionaron para el análisis aquellos taxa que estaban presentes en más de un $50 \%$ tanto de las muestras de polen como en los censos de vegetación. Estos taxa correspondieron a tipo Senecio, subfamilia Papilionoideae, familia Poaceae y Mulinum spinosum. Dentro del tipo Senecio se reunieron los granos de polen de Compositae Tubuliflorae con espinas entre 2 y 5 $\mu \mathrm{m}$ de longitud (Senecio, Baccharis y Chrisanthemoides), los que se diferenciaron de los granos de los géneros Gnaphalium, Nassauvia y Chuquiraga que poseen espinas de menor longitud. En los censos de vegetación se verificó la presencia de las compuestas Senecio, Baccharis y Chrisanthemoides, las que fueron agrupadas en tipo Senecio para relacionarlo con el polen. Los taxa correspondientes a la subfamilia Papilionoideae, y los géneros Adesmia, Astragalus y Anarthrophyllum, presentes en los censos de vegetación, se agruparon dentro de la subfamilia Papilionoideae para permitir su comparación con el polen que no pudo ser determinado para categorías inferiores. Los géneros Stipa, Poa, Hordeum, Festuca y Bromus, presentes en la vegetación, fueron incluidos dentro de la familia Poaceae durante el análisis de polen. En el caso 
de Mulinum spinosum fue posible realizar un análisis a nivel específico tanto en términos de la vegetación como en el polen.

Se realizaron análisis de regresión simple utilizando el programa BMDP New System (1994). Las regresiones se efectuaron entre el porcentaje de polen y el porcentaje de cobertura de vegetación para cada taxón. Las variables dependientes (polen) e independientes (cobertura de vegetación) fueron linealizadas mediante su elevación a la potencia 0,25 , excepto tipo Senecio que se elevó a la potencia 0,5 . En todos los casos este procedimiento se realizó de acuerdo a los mejores índices de correlación obtenidos (coeficiente de correlación $r$ de Pearson). Se calcularon los valores residuales estandarizados, se seleccionó el modelo según el cual un gráfico entre los valores residuales no mostrara un patrón fijo de variación, y se obtuvo el valor t de Student. Asimismo, se calcularon los límites de confianza del $95 \%$ para el origen y la pendiente.

\section{RESULTADOS Y DISCUSIÓN}

La Tabla 1 muestra los resultados obtenidos del análisis de regresión para cada uno de los taxa seleccionados. El valor predictivo de la regresión entre el polen y la vegetación está determinado por: (1) la magnitud de la correlación entre las variables (valor de r), (2) la distancia entre el intercepto de la recta sobre la ordenada y el origen (a), y (3) por el valor de la pendiente (b).

Las mejores correlaciones entre los tipos polínicos estudiados y los porcentajes de cobertura de vegetación se obtuvieron para el tipo Senecio y para Mulinum spinosum. Esto indica una correspondencia fuerte entre el porcentaje de cobertura vegetal y el porcentaje de polen para los dos taxa, lo que permite estimar el porcentaje de polen a partir de la cobertura vegetal en el $81 \%$ de los casos. Por otro lado, las correlaciones para Poaceae y Papilionoideae fueron menores con el 66 y $61 \%$ de los casos, respectivamente. La intersección de la recta de regresión con la ordenada para los cuatro taxa, fue mayor que cero, lo que se interpreta como presencia de polen regional.

El análisis de la familia Poaceae (Fig. 2a) muestra que los cambios en el porcentaje de la cobertura vegetal se traducen en cambios en el porcentaje de polen, en una proporción 1:0,18 (valor b de la Tabla 1). El valor del intercepto es alto $(\mathrm{a}=$

TABLA 1

Resultados obtenidos del análisis de regresión simple entre cobertura vegetal y polen para la familia Poaceae, Mulinum spinosum, tipo Senecio y subfamilia Papilionoideae

Results obtained from regression analysis between plant cover and pollen for family Poaceae, Mulinum spinosum, Senecio-type, and subfamily Papilionoideae

\begin{tabular}{ll}
\hline Taxón & $\begin{array}{l}\text { Ecuación de la recta } \\
(\mathrm{y}=\mathrm{a}+\mathrm{bx})\end{array}$ \\
\hline Poaceae & $\mathrm{a}=1,8307(1,4467 ; 2,2146)$ \\
& $\mathrm{b}=0,1809(0,0241 ; 0,3376)$ \\
& $\begin{array}{l}\text { Pearson } \mathrm{r}=0,6564 \\
(\% \text { Polen })^{0.25}=1,8307+0,1809(\% \text { Cobertura vegetal })^{0.25}\end{array}$ \\
& $\mathrm{a}=0,6185(0,1183 ; 1,1188)$ \\
& $\mathrm{b}=0,6278(0,2920 ; 0,9636)$ \\
& $\begin{array}{l}\text { Pearson } \mathrm{r}=0,8156 \\
(\% \text { Polen })^{0.25}=0,6185+0,6278(\% \text { Cobertura vegetal })^{0.25}\end{array}$ \\
& $\mathrm{a}=1,7224(0,5759 ; 2,8690)$ \\
& $\mathrm{b}=0,6667(0,3030 ; 1,0303)$ \\
& $\begin{array}{l}\text { Pearson } \mathrm{r}=0,8102 \\
(\% \text { Polen })^{0.50}=1,7224+0,6667(\% \text { Cobertura vegetal })^{0.5}\end{array}$ \\
& $\mathrm{a}=0,2393(-0,2179 ; 0,6975)$ \\
& $\mathrm{b}=0,3141(0,0072 ; 0,6210)$ \\
& $\begin{array}{l}\text { Pearson } \mathrm{r}=0,6109 \\
\left.(\% \text { Polen })^{0.25}=0,2393+0,3141 \text { (\% Cobertura vegetal }\right)^{0.25}\end{array}$ \\
\hline
\end{tabular}

Los valores entre paréntesis corresponden a intervalos para el $95 \%$ de confianza 
Poaceae

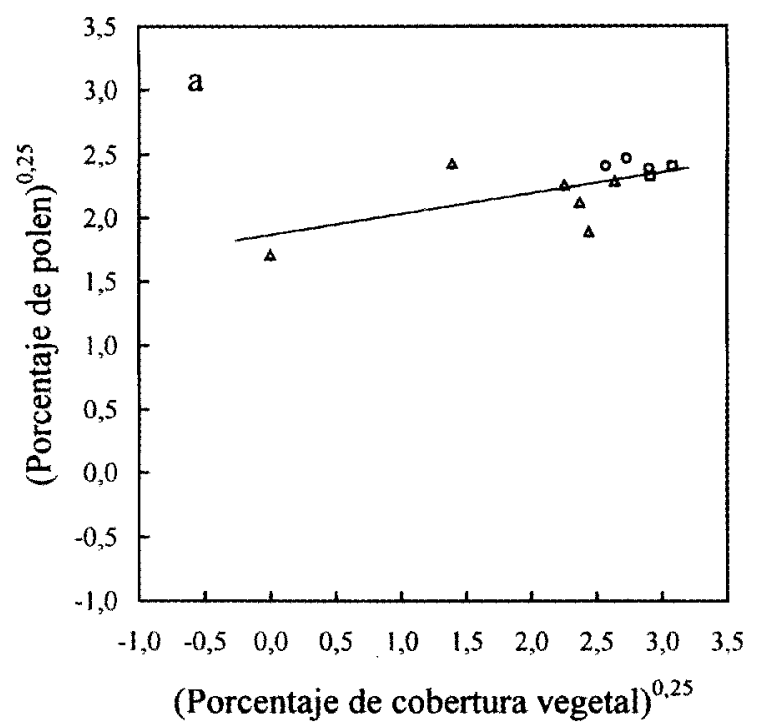

tipo Senecio

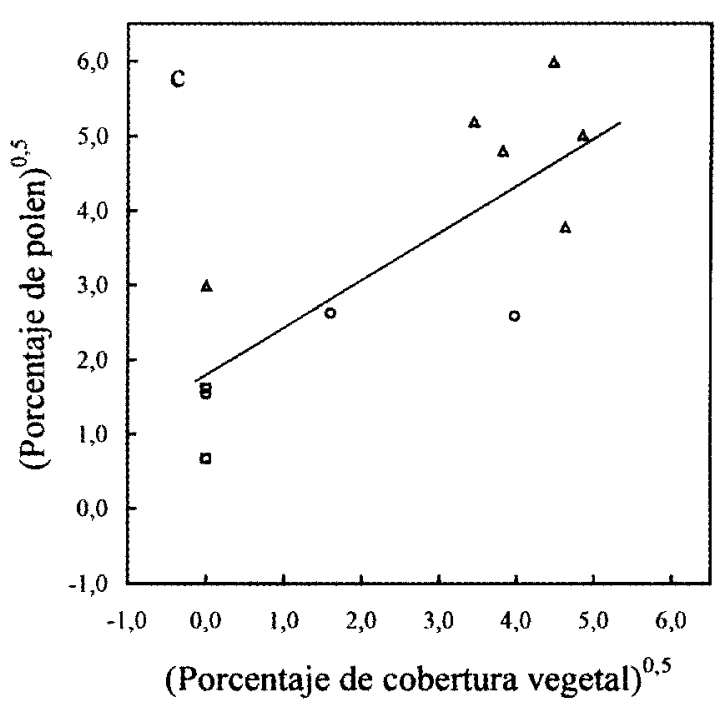

Mulinum spinosum

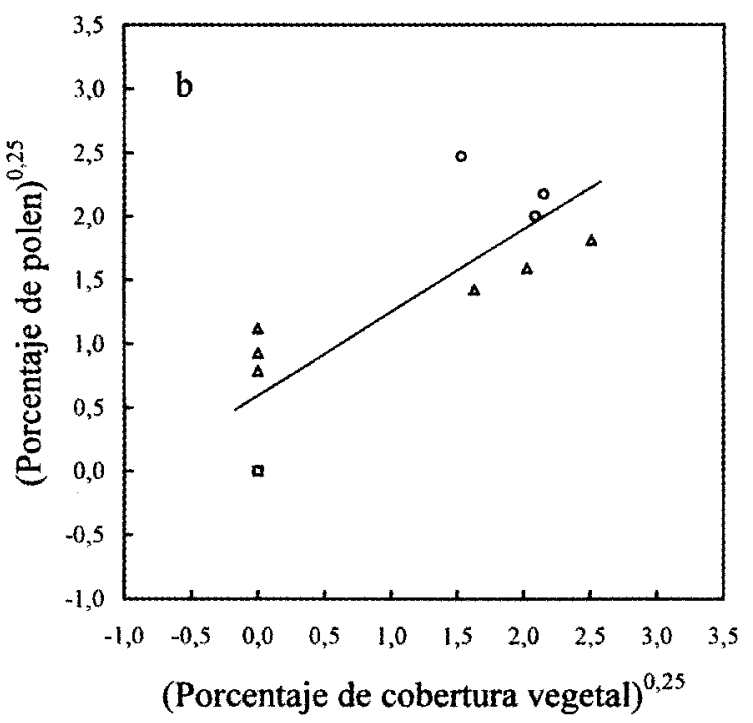

Papilionoideae

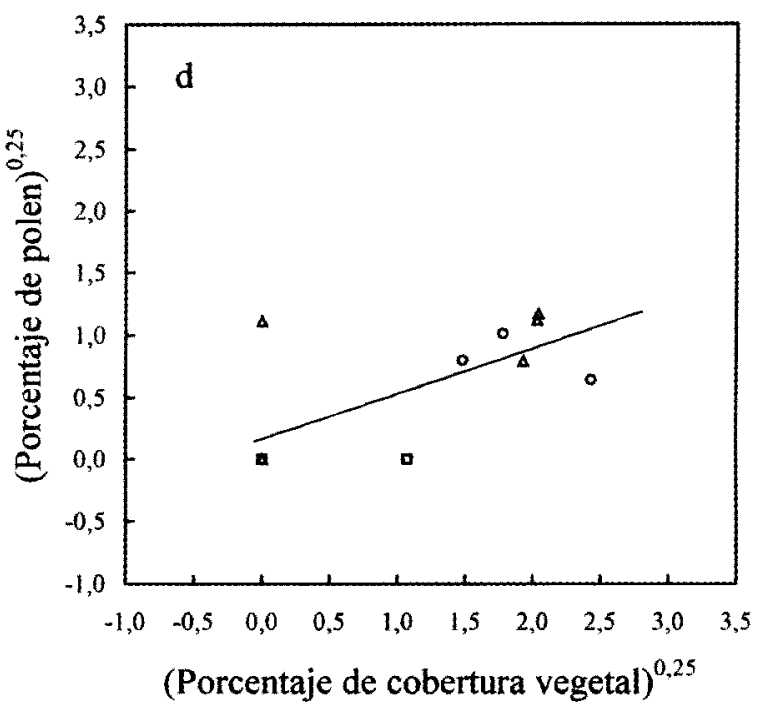

Fig. 2: Regresión lineal simple entre el porcentaje de cobertura vegetal y el porcentaje de polen para (a) la familia Poaceae, (b) Mulinum spinosum, (c) tipo Senecio y (d) la subfamilia Papilionoideae. Datos transformados a la potencia 0,25 , excepto para tipo Senecio que están transformados a la potencia 0,5 . Las estaciones corresponden a los distritos central $(\Delta)$, distrito occidental $(O)$ y subandino $(\square)$.

Simple lineal regression between percentage of plant cover and pollen percentage for (a) family Poaceae,( b) Mulinum spinosum, (c) Senecio-type, and (d) subfamily Papilionoideae. Data were transformed to the 0.25 power, except for Senecio-type, whose data were transformed to the 0.50 power. Central district $(\Delta)$, occidental district $(O)$, and subandean district ( $\square$ ).

1,83); se registra aproximadamente un $10 \%$ de polen (valor a sin transformar $=1,83^{4}$ ) en ausencia de vegetación del taxón. La pendiente de la recta es baja, lo que sugiere que el porcentaje de polen no está fuertemente asociado a la cobertura vegetal. Es probable que la abundancia del polen dependa no solo la vegetación local sino también de un aporte regional. La familia Poaceae se encuentra bien representada en los distritos subandino, occidental y central (Fig. 3a). Las muestras de los distritos subandino y occidental se presentan agrupadas en la Fig. 2a, donde una 
alta cobertura vegetal está asociada a un alto porcentaje de polen. Esto es, existe una relación estrecha entre el polen hallado en el sedimento superficial y la fuente de emisión. Sin embargo, en el distrito central hay gran variación de la cobertura de vegetación respecto del porcentaje de polen; ello sería producto del aporte de polen hacia este sector por parte de los distritos subandinos y occidental como resultado de los vientos predominantes del oeste.

En regiones áridas los vientos juegan un papel muy importante, principalmente debido a que los vientos son intensos y pueden abarcar varios miles de kilómetros con pocas barreras en su camino. Estos vientos juegan un rol importante sobre el espectro polínico (Horowitz 1992). Además del polen producido por la vegetación local árida, los espectros polínicos incluyen polen que es arrastrado y depositado por el viento desde zonas más húmedas. Los vientos que llegan a las zonas semidesérticas desde regiones más húmedas pueden llevar consigo polen que formará parte de los sedimentos de aquellas y pueden, debido al bajo factor de enmascaramiento de la flora polínica de tierras secas, crear una falsa impresión de ser una vegetación mucho más rica que la que en realidad crece en las zonas semiáridas estudiadas.

En el caso de Mulinum spinosum (Fig. 2b), las variaciones en el porcentaje de la cobertura vegetal se corresponden con las variaciones en el porcentaje de polen en una proporción de 1:0,6 (valor b de la Tabla 1). Por consiguiente, la depositación está relacionada con la producción local y hay una alta asociación entre los porcentajes de cobertura vegetal y de polen. Por otra parte, el aporte de polen regional de este taxón no es importante; se verifica sólo un $0,15 \%\left(0,62^{4}\right)$ de polen en ausencia de Mulinum spinosum en la vegetación. Esta especie es característica del distrito occidental (Fig. 3b) y las muestras correspondientes a este distrito son las que tienen la mejor representación de polen y vegetación. Mulinum spinosum también aparece en el distrito central, pero no es una especie característica de éste; las muestras se reúnen en dos grupos, uno con ausencia de cobertura vegetal y presencia de polen transportado desde el oeste, y otro, con presencia de polen y vegetación. Tanto en los censos de vegetación como en las muestras de polen del distrito subandino no hay Mulinum spinosum.

El tipo Senecio (Fig. 2c) muestra una relación entre el porcentaje de cobertura vegetal y el porcentaje de polen de 1:0,7 (valor b de la Tabla 1), la más alta de los taxa incluidos en este estudio. Por lo tanto, la cobertura vegetal integrada por el tipo Senecio se asocia en un alto grado con el porcentaje de polen; el modelo muestra que hay aproximadamente un $3 \%\left(1,72^{2}\right)$ de polen en ausencia de vegetación. Los géneros incluidos en este grupo (Senecio y Baccharis) son característicos de los distritos central y occidental (Fig. $3 c)$. En el central se observan los valores más altos de polen y de vegetación, mientras que en el occidental aparece este grupo con menor porcentaje de cobertura y de polen. En el distrito subandino se detectó la presencia de polen en ausencia de cobertura de estos géneros.

En cuanto a la subfamilia Papilionoideae (Fig. $2 \mathrm{~d}$ ), la relación entre el porcentaje de cobertura vegetal y el porcentaje de polen es de 1:0,31 (valor b de la Tabla 1), lo que indica que el porcentaje de cobertura vegetal no refleja adecuadamente el porcentaje de polen. Por otro lado, el intercepto es bajo, lo que sugiere que existe un bajo aporte de polen regional. Se detectó sólo un $0,003 \%\left(0,24^{4}\right)$ de polen en ausencia de vegetación de la subfamilia Papilionoideae. Esto se podría explicar por la polinización zoófila que presenta este grupo, evidenciada por la baja densidad de polen que aparece en la lluvia polínica.

Al comparar la subfamilia Papilionoideae con la familia Poaceae se observa que la cobertura de la vegetación está poco asociada con el porcentaje de polen, pero que en el caso de Papilionoideae no hay un aporte de polen regional o local en el sedimento superficial. La presencia de este taxón no ha sido importante en las muestras de polen ni en los censos de vegetación (Fig. 3d) a diferencia de los demás taxa estudiados.

En los cuatro taxa analizados Poaceae, tipo Senecio, Mulinum spinosum y Papilionoideae se halló un buen ajuste de las rectas de regresión para polen-vegetación. El análisis de regresión entre datos actuales de vegetación y polen permite conocer la relación cuantitativa que existe entre ellos. Se considera el polen como variable dependiente y la vegetación como variable independiente; para un índice de correlación alto la variable independiente podrá predecir con un determinado porcentaje de probabilidad los valores de la dependiente. Este análisis da como resultado una ecuación de regresión que describe esta relación, aplicable a estudios de historia de la vegetación, cuando sólo se dispone de datos de polen fósil. El conocimiento de esta relación permitirá utilizar a la variable dependiente para predecir la independiente. Así, se podrá describir la vegetación del pasado perteneciente al taxón estudiado.

Se concluye que el análisis de regresión, empleando datos de polen y vegetación actuales, es una herramienta útil en la interpretación de datos polínicos fósiles y en la descripción de la vegeta- 
Poaceae

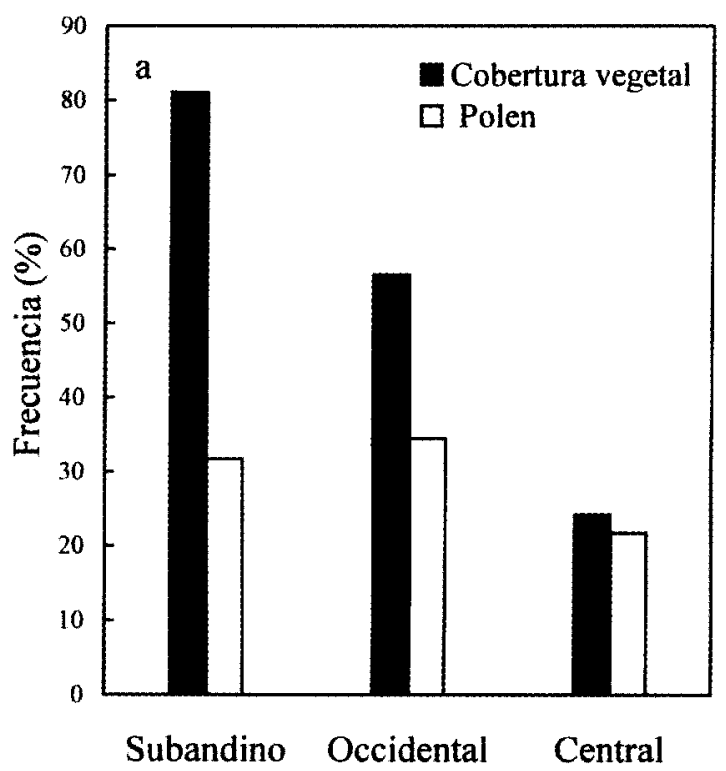

tipo Senecio

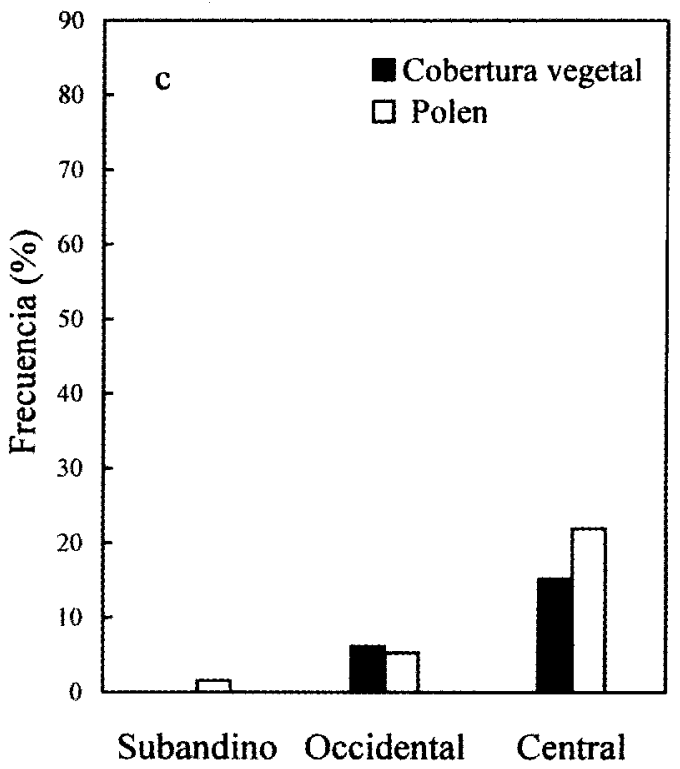

Mulinum spinosum

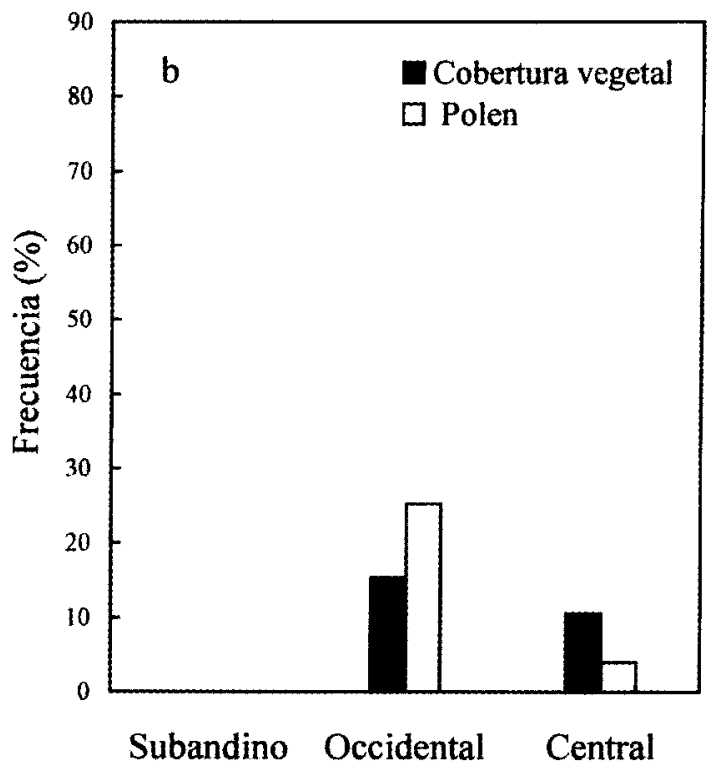

Papilionoideae

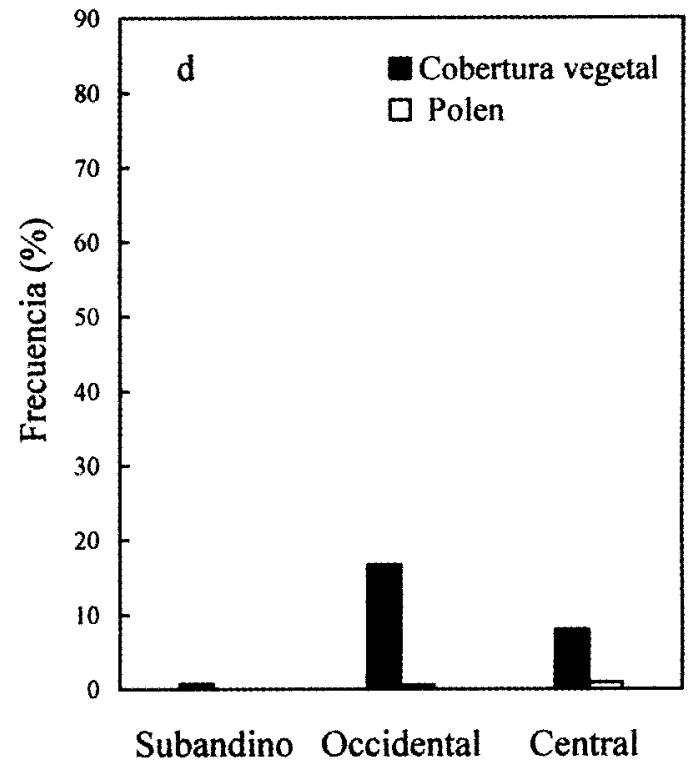

Fig. 3: Porcentaje de cobertura vegetal y de polen superficial en el distrito subandino, distrito occidental y distrito central para (a) la familia Poaceae, (b) Mulinum spinosum, (c) tipo Senecio y (d) la subfamilia Papilionoideae.

Percentage of vegetation cover and superficial pollen in the subandean, occidental, and central districts for (a) family Poacae, (b) Mulinum spinosum, (c) Senecio-type, and (d) subfamily Papilionoideae.

ción del pasado. Las ecuaciones de regresión obtenidas a partir de este estudio representan modelos que explican las relaciones entre el polen y la vegetación del área del sur de Chubut, a $\operatorname{los} 45^{\circ} \mathrm{S}$ y entre los $68^{\circ} 57^{\prime}$ y $\operatorname{los} 71^{\circ} 26^{\prime} \mathrm{O}$. Estos modelos permitirán predecir, con distintos nive- les de confianza, la abundancia de vegetación de los taxa Poaceae, Mulinum spinosum, el tipo Senecio y la subfamilia Papilionoideae en el pasado a partir de polen fósil para el área estudiada. Sin embargo, debido a que las relaciones entre polen y vegetación son dependientes de factores 
ecológicos, y adquieren distintos valores en diferentes regiones climáticas, se requiere de estudios adicionales sobre relaciones polen-vegetación a distintas escalas espaciales de modo que las predicciones hechas por los modelos sean más confiables.

\section{AGRADECIMIENTOS}

Queremos expresar que este trabajo debería llevar tanto a Trivi como a Burry como primeras autoras, pero el formato de la revista no lo permite. Apreciamos las sugerencias y lectura crítica del manuscrito por parte de Héctor L. D'Antoni así como su generoso aporte de ideas. También agradecemos a quienes nos acompañaron y ayudaron en nuestro trabajo de campo: Máximo Magnoni, Julio Mandri y Visnja Mavrek. Este trabajo se realizó con un subsidio otorgado por la Universidad Nacional de Mar del Plata.

\section{LITERATURA CITADA}

ANDERSEN ST (1970) The relative pollen productivity and pollen representation of north European trees, and correction factors for tree pollen spectra determined by surface pollen analyses from forests. Danmarks Geologiske Undersøgelse Series II 96: 1199.

BEESKOW AM, HE DEL VALLE \& CM ROSTAGNO (1987) Los sistemas fisiográficos de la región árida y semiárida de la Provincia de Chubut. Secretaría de Ciencia y Técnica, Delegación Regional Patagónica, Puerto Madryn, Argentina. 145 pp.

BMDP New System for Windows (1994), version 1. BMDP Statistical Software, Inc., Los Angeles, California.

BRADSHAW RHW (1981) Modern pollen-representation factors for woods in south-east England. Journal of Ecology 69: 45-70.

BRADSHAW RHW \& T WEBB III (1985) Relationships between contemporary pollen and vegetation data from Wisconsin and Michigan, USA. Ecology 66: 721-737.

CABRERA AL (1994) Regiones fitogeográficas argentinas. Enciclopedia argentina de agricultura y jardinería. Primera reimpresión. Tomo $2.85 \mathrm{pp}$.

CANFIELD R (1941) Application of the line interception method in sampling range vegetation. Journal of Forestry 39: 388- 394.

CURTIS JT (1959) The vegetation of Wisconsin: an ordination of plant communities. University Wisconsin Press, Madison, Wisconsin. 657 pp.

D'ANTONI HL (1990) La importancia del análisis de polen en la reconstrucción del clima y la vegetación del pasado. Monografía de la Academia Nacional de Ciencias Exactas Físicas y Naturales (Buenos Aires) 5: 106-113.
D'ANTONI HL (1991) Modern pollen dispersal in southern Argentina. Bamberger Geographische Schriften 11: 209-227.

D'ANTONI HL \& F SCHÄBITZ (1995) Remote sensing and Holocene vegetation: history of global change. World Resource Review 7: 282-288.

D'ANTONI HL \& MA SPANNER (1993) Remote sensing and modern pollen dispersal in southern Patagonia and Tierra del Fuego (Argentina): models for palaeoecology. Grana 32: 29-39.

DAVIS MB (1963) On the theory of pollen analysis. American Journal of Science 261: 897-912.

DAVIS MB \& JC GOODLETT (1960) Comparison of the present vegetation with pollen-spectra in surface samples from Brownington pond, Vermont. Ecology 41: 346-357.

ERDTMAN G (1943) An introduction to pollen analysis. Chronica Botanica, Waltham, Massachusetts. $238 \mathrm{pp}$.

FAEGRI K \& J IVERSEN (1989) Textbook of pollen analysis. Fourth edition. John Wiley \& Sons, New York. $328 \mathrm{pp}$.

FAGERLIND F (1952) The real signification of pollen diagrams. Botaniska Notiser (Sweden) 105: 185-224.

GODWIN H (1934a) Pollen analysis: an outline of the problems and potentialities of the method. I. Technique and interpretation. New Phytologist 33: 278-305.

GODWIN H (1934b) Pollen analysis: an outline of the problems and potentialities of the method. II. General applications of pollen analysis. New Phytologist 33: 325-358.

GRAY J (1965) Extraction techniques. En: Kummel B \& D Raup (eds) Handbook of paleontological techniques. Part III. Techniques in palynology: 530-587. W.H. Freeman \& Co., San Francisco, California.

HOROWITZ A (1992) Palynology of arid lands. Elsevier Science Publishers, Amsterdam, The Netherlands. $546 \mathrm{pp}$.

JACKSON ST (1994) Pollen and spores in Quaternary lake sediments as sensors of vegetation composition: theoretical models and empirical evidence. En: Traverse A (ed) Sedimentation of organic particles: 253-286. Cambridge University Press, Cambridge, United Kingdom.

JACKSON ST, T WEBB III, IC PRENTICE \& JE HANSEN (1995) Exploration and calibration of pollen/ vegetation relationships: a PC program for the extended R-value models. Review of Paleobotany and Palinology 84: 365-374.

JANSSEN CR (1970) Problems in the recognition of plant communities in pollen diagrams. Vegetatio (The Netherlands) 20: 187-198.

MANCINI MV (1989) Depositación del polen actual en el sur de Santa Cruz. Tesis de Doctorado, Universidad Nacional de Mar del Plata, Facultad de Ciencias Exactas y Naturales, Mar del Plata, Argentina. 124 pp.

MUELLER-DOMBOIS D \& H ELLENBERG (1974) Aims and methods of vegetation ecology. John Wiley \& Sons, New York, New York. 547 pp.

PÁEZ MM (1991) Palinología de Campo Moncada 2 (Chubut): interpretación paleoecológica para el Holoceno. Tesis de Doctorado, Universidad Nacional de La Plata, Facultad de Ciencias Naturales y Museo, La Plata, Argentina. 275 pp. 
PARSONS RW \& IC PRENTICE (1981) Statistical approaches to $R$-values and the pollen vegetation relationship. Review of Palaeobotany and Palynolology 32: 127-152.

PRENTICE IC (1986) Forest-composition calibration of pollen data. En: Berglund BE (ed) Handbook of Holocene palaeoecology and palaeohydrology: 799816. John Wiley \& Sons, Chichester, New York.

SORIANO A (1956) Los distritos florísticos de la provincia patagónica. Revista de Investigaciones Agrícolas (Argentina) 10: 321- 357.

STOKMARR J (1971) Tablets with spores used in absolute pollen analysis. Pollen et Spores (Paris) 13: 615-621.
VON POST L (1918) Skosträdpollen y sydsvenska torvmosslagerföljder. Forh. 16. skand. naturforskermote 1916: 433-465. Pollen et Spores (Paris) 9: 376-401. (Traducido por Davis MB \& K Faegri).

VON POST L (1967) Forest tree pollen in south Swedish peat bog deposits. Pollen et Spores (Paris) 9: 375401.

WALKER D (1972) Quantification in historical plant ecology. Proceedings of the Ecological Society of Australia 6: 91-104.

WEBB III T, SE HOWE, RHW BRADSHAW \& KM HEIDE (1981) Estimating plant abundances from pollen percentages: the use of regression analysis. Review of Palaeobotany and Palynology 34: 269-300.

Editor Asociado: C. Villagrán

Recibido el 28 de mayo de 1998; aceptado el 20 de septiembre de 2000 